\title{
Bargaining over Fair Channel Sharing between Wi-Fi and LTE-U Networks
}

\author{
Andrey Garnaev ${ }^{1,2}$, Shweta Sagari ${ }^{2}$, and Wade Trappe ${ }^{2}$ \\ 1 Saint Petersburg State University, St Petersburg, Russia \\ garnaev@yahoo.com \\ 2 WINLAB, Rutgers University, North Brunswick, USA \\ shsagari@winlab.rutgers.edu, trappe@winlab.rutgers.edu
}

\begin{abstract}
Wireless networks are increasingly moving towards a heterogeneous operating model involving the sharing of spectrum resources by different access technologies. Sharing wireless resources between different wireless technologies requires protocols that share spectrum in an equitable manner. In this paper, we examine the time-sharing of wireless channels by Wi-Fi and LTE-U networks. To design fair access protocol for the networks we use $\alpha$ fairness criterion. It allows to find a continuum of fair protocols (a protocol per $\alpha$ ). To find the most fair from this continuum of fair protocols we apply Nash bargaining approach. In particular, we show that such a time-sharing bargaining protocol, in spite of the interference between signals, can lead to a gain for both networks under an increase of the transmission power to one of them.
\end{abstract}

Key words: Wi-Fi, LTE-U, Fairness, Bargaining

\section{Introduction}

The demand for radio spectrum has been dramatically increasing over the last decade, in large part due to the emergence of new wireless applications and devices. In order to address the increased demand on congested bands of radio spectrum, there have been numerous proposals to make under-utilized spectrum bands open for simultaneous sharing by different wireless technologies. However, allowing different wireless access networks, such as cellular and wireless local area networks, to operate in proximity to each other while in the same frequency bands, requires the development of new technologies that can coordinate sharing and coexistence. In fact, the significant value that spectrum sharing can provide to technologies like LTE-U (LTE in unlicensed spectrum) and Wi-Fi has led to prominent discussions occurring in the standards community (notably the 3rd Generation Research Project, i.e. 3GPP, community) regarding the throughput and latency requirements needed for maintaining fair coexistence [1] even though the full specification of LTE-U has not been finalized.

The literature describing the coexistence between LTE-U and Wi-Fi technologies is very recent and thus there are only a few works investigating Wi-Fi and LTE-U coexistence. In [2], simulation analysis was used to show that LTE 
system performance can be slightly affected by coexistence, while Wi-Fi performance is significantly impacted by LTE transmissions. In [3], it was pointed out that the coexistence of LTE and Wi-Fi must be carefully investigated since, as it was previously illustrated, Wi-Fi networks might be severely impacted by LTE transmissions. The performance of coexisting femtocell and Wi-Fi networks operating over a fully-utilized, unlicensed band were analytically modeled in [4]. LBT mechanisms for LAA-LTE was suggested in [5] to ensure that it operates at least as fairly as Wi-Fi in unlicensed spectrum. The effects of Wi-Fi channel access parameters on the performance of Wi-Fi and Femtocell networks were investigated in [6]. A novel system to support co-existence between Wi-Fi and LTE was developed in [7] allowing to decode the interfering signals under cross technology interference even when the interfering signals have similar power. A traffic-balancing algorithm was explored in [8], while a fair and QoS-based unlicensed spectrum splitting strategy between Wi-Fi and femtocell networks was suggested in [9]. Results of experimental evaluation on the coexistence between Wi-Fi and LAA-LTE were presented in [10]. A proportional fair allocation scheme for Wi-Fi and LTE coexistence was develop in [11]. Modeling the coexistence of LTE and Wi-Fi heterogeneous networks was performed in [12].

A goal of this paper is to design the most fair channel access protocol for a channel shared between LTE-U and Wi-Fi networks. The model studied in the paper is based on the model for coordinated dynamic spectrum management between LTE-U and Wi-Fi networks suggested in [13] and [14]. Namely, in [13], a system for coordinating between multiple heterogeneous networks to improve spectrum utilization and facilitate co-existence, which involves building a logically-centralized spectrum management controller using the principles of a Software Defined Networking was outlined. Based on this architecture, an optimization model to maximize the aggregated Wi-Fi+LTE throughput was designed and tested in [14]. This optimization problem was divided into two steps: in the first step, based on information about the networks and their users, power control optimization problems were solved to obtain the optimal throughput for only-Wi-Fi access, and for joint Wi-Fi+LTE access to the channels. In the second step, based on these throughputs, a maxmin problem involving timedivision access between the different access technologies to the shared resources, was solved numerically. Later, in [15], an analytical solution for the family of $\alpha$ fair protocols (which includes also the maxmin protocol) for shared access to the channels with equal priority of the networks was given. The evaluation of such joint coordination led to the important insight that such a dual optimization approach can actually increase the aggregated Wi-Fi+LTE throughput compared to each technology separately.

One critical challenge, though, facing the design of a system trying to support fairness between two or more parties is the selection of an appropriate fairness criterion. Even for a single criterion, such as maxmin, the resulting fair allocation strategies can be complicated, but the question of whether that was the right criterion is rarely, if ever, addressed. The matter becomes more complex, though, when one considers a continuum of fairness criteria, such as represented by the 
family of $\alpha$-fairness schemes, and which of those resulting allocation strategies should be chosen. In this paper, we examine this problem for a communication scenario involving the fair time-division allocation between LTE-U and Wi-Fi communication systems, and arrive at an approach that allows a system engineer to chose the unique optimal protocol from a continuum of optimal protocols (i.e. an optimal protocol per $\alpha$ ). Namely, we show that the Nash bargaining solution over this continuum of protocols can be considered as the optimal access protocol for the shared resource from point of view of both networks. We solve the problem in closed form, which allows us to illustrate the improved efficiency possible by both technologies jointly accessing the shared resource.

The organization of this paper is as follows: in Section 2, the basic interference model between Wi-Fi and LTE that we use is described. In Section 3, the fair time-division access to a shared channel is formulated and solved in closed form. Also, it is illustrated that for each of the participating networks, there is generally no monotonic dependence in benefit associated with an increase in the $\alpha$ fairness coefficient. Thus, a question arises: out of the associated continuum of optimal fairness access protocols, which one should be considered the optimal one. To answer these questions, in Section 4, auxiliary properties of the optimal solution are obtained. This has allowed us, in Section 5, to apply Nash bargaining to find the best trade-off access protocol for both networks. Finally, in Section 6, a discussion of the results is offered, and in Appendix, sketch of proofs is given.

\section{Model of interference between Wi-Fi and LTE}

We begin by briefly describing a simple analytical model suggested in [14] for the interference between Wi-Fi and LTE, which will be used as a basic model for numerical illustration in this paper. In this model both technologies utilizing the same amount of bandwidth (e.g. $20 \mathrm{MHz}$ ) share a single spectrum channel. Also, we assume that there is only a single Wi-Fi network and a single LTE-U cell operating on that channel, and hence we do not worry about problems involving clients choosing between multiple operators within a specific technology. Let the transmission power for the Wi-Fi and LTE cell be $p_{W}$ and $p_{L}$. Then, a model for the throughput, $R_{i}, i \in\{W, L\}$ is given as

$$
R_{i}=\alpha_{i} B \log _{2}\left(1+\beta_{i} h_{i i} p_{i} /\left(N_{i}+h_{i j} p_{j}\right)\right), i, j \in\{W, L\}, i \neq j,
$$

where $B$ is the channel bandwidth, $h_{i j}$ are channel gains, $N_{i}$ is noise power for receiver $i, \beta_{i}$ is a factor associated with the modulation scheme, $\alpha_{i}$ is bandwidth efficiency, namely, for Wi-Fi (i.e., $i=W$ ) it is the bandwidth efficiency of CSMA/CA, while for LTE (i.e., $i=L$ ) it is the bandwidth efficiency resulting from factors such as adjacent channel leakage and practical filters design.

For coordinating between units of such multiple heterogeneous networks to improve spectrum utilization and facilitate co-existence, a Global Controller (GC) was proposed[14]. The GC receives information from each network's operating conditions in order to forecast the throughput under separate or joint access by the Wi-Fi and LTE networks to the channel. In this paper, we assume 
that such throughput values have been obtained and focus our research on the problem of fair access of the networks to the channels. We note that there is extensive work being done by the community on facilitating such information sharing.

\section{Fair Time Division Access Optimization}

In this Section, we formulate the problem of determining the fraction of time that each wireless technology (Wi-Fi and LTE-U) accesses the channel so as to fairly coexist. Following [14], we assume that the total throughput of each network is proportional to the fraction of time that technology uses the channel, and that it also depends on whether the channel access is simultaneous or not. In the considered scenario, Wi-Fi has priority to access the channels, i.e., Wi-Fi always has access, while LTE-U may or may not have access. To describe the problems let us introduce the following notations:

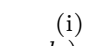

(i) $q^{W}$ is the fraction of time the channel is accessed by the Wi-Fi network only (Wi-Fi access

(ii) $q$ is the fraction of tine the channel is accessed by both the networks simultaneously (joint $W i$-Fi and LTE-U access mode);

(iii) Without loss of generality, we can assume that the total time duration for access to the channels is $[0,1]$. Thus, $q^{W}+q=1$, and the vector of time fractions $\boldsymbol{q}=\left(q, q^{W}\right)$ can be interpreted also as a probability vector. Let $\boldsymbol{Q}$ be the set of all such vectors;

(iv) $R^{W}$ is the throughput of Wi-Fi network per time unit, when the network is in Wi-Fi access mode;

(v) $R_{W}^{L}$ and $R_{L}^{W}$ are respectively the throughputs for LTE-U and Wi-Fi networks per time unit, when operating in joint $\mathrm{Wi}-\mathrm{Fi}$ and LTE-U access mode, where both networks access the channel simultaneously. It is natural to assume that extra interference in the network reduces its throughput, i.e., $R_{L}^{W} \leq R^{W}$

(vi) $T^{W}$ is the total throughput of the Wi-Fi network, i.e., $T^{W}(\boldsymbol{q})=q^{W} R^{W}+q R_{L}^{W}$;

(vii) $T^{L}$ is the total throughput of LTE-U network, i.e., $T^{L}(\boldsymbol{q})=q R_{W}^{L}$.

If $q=0$, we call such strategy $\boldsymbol{q}$ as a channel on/off strategy, i.e., when the networks do not jointly access the channels simultaneously, but only one does. If $q>0$, we call such a strategy $\boldsymbol{q}$ as a channel sharing strategy, i.e., when the networks might access the channel simultaneously. As an aside remark, we note that different resource sharing strategies could be applied for different network optimization problems, see for example, channel sharing [16], bandwidth scanning [17], spectrum coexistence [18], time sharing [19], and channel sharing in selfish transmission for low SNR mode [20] and general SNR mode [21].

When allocating the fraction of time for these access modes, the issue of fairness naturally arises as improving one system's throughput typically won't lead to improvement in the other system. A survey of different fairness concepts used in wireless communication is given in [22]. Generally, in the fairness problem, there are $n$ agents and each of them has an utility that depend on its share of a common resource. The problem then involves allocating such shares fairly between the agents. Of course, the result depends on the criterion for fairness, and maxmin is one such criterion that is popular. We focus though on $\alpha$-fairness, which allows one to consider, in a uniform scale, such separate fairness concepts as bargaining (for $\alpha=1$ ) and maxmin (for $\alpha$ tending to infinity). In [23], a general problem for fair throughput assignment ( $\alpha$-fairness) was suggested. In [24], 
a problem of bargaining over the fair trade-off between secrecy and throughput in OFDM communications was solved. In [25], in the context of LTE-A networks, cooperative bargaining solutions for resource allocation over the available component carriers was investigated as well as the optimal tradeoff between fairness and efficiency, which allows one to select the most appropriate solution over all of the available carriers. In [26], bargaining problem over fair performing dual radar and communication task was solved. In [27], fair power control was applied for resources allocation by base station under uncertainty. In [28], a Baysian game of fair power allocation is solved.

In our scenario, the $\alpha$-fair channel access problem for LTE-U and Wi-Fi networks to a shared channel, we consider the expected throughput of networks as utilities. Then, the $\alpha$-fair channel access protocol $\boldsymbol{q}_{\alpha}$ is given as solution of the following problem:

$$
\text { maximize } v_{\alpha}(\boldsymbol{q}) \text {, subject to } \boldsymbol{q} \in \boldsymbol{Q}
$$

with

$$
\begin{aligned}
v_{\alpha}(\boldsymbol{q}) & = \begin{cases}\left(T^{W}(\boldsymbol{q})\right)^{1-\alpha} /(1-\alpha)+\left(T^{L}(\boldsymbol{q})\right)^{1-\alpha} /(1-\alpha), & \alpha \neq 1, \\
\ln \left(T^{W}(\boldsymbol{q})\right)+\ln \left(T^{L}(\boldsymbol{q})\right), & \alpha=1,\end{cases} \\
& = \begin{cases}\frac{\left(q^{W} R^{W}+q R_{L}^{W}\right)^{1-\alpha}}{1-\alpha}+\frac{\left(q R_{W}^{L}\right)^{1-\alpha}}{1-\alpha}, & \alpha \neq 1, \\
\ln \left(q^{W} R^{W}+q R_{L}^{W}\right)+\ln \left(q R_{W}^{L}\right), & \alpha=1 .\end{cases}
\end{aligned}
$$

Theorem 1 The optimal $\alpha$-fair strategy $\boldsymbol{q}_{\alpha}$ is given as follows:

$$
\begin{gathered}
q_{\alpha}^{W}= \begin{cases}0, & R_{W}^{L}\left(R_{L}^{W} / R_{W}^{L}\right)^{\alpha}+R_{L}^{W} \geq R^{W}, \\
1-\frac{\frac{R_{L}^{W}}{R^{W}-R_{L}^{W}}\left(\frac{R_{W}^{L}}{R^{W}-R_{L}^{W}}\right)^{1 / \alpha-1}}{1+\left(\frac{R_{W}^{L}}{R^{W}-R_{L}^{W}}\right)^{1 / \alpha-1}}, & R_{W}^{L}\left(R_{L}^{W} / R_{W}^{L}\right)^{\alpha}+R_{L}^{W}<R^{W},\end{cases} \\
q_{\alpha}= \begin{cases}1, & R_{W}^{L}\left(R_{L}^{W} / R_{W}^{L}\right)^{\alpha}+R_{L}^{W} \geq R^{W}, \\
\frac{R^{W}}{R^{W}-R_{L}^{W}} \frac{\left(\frac{R_{W}^{L}}{R^{W}-R_{L}^{W}}\right)^{1 / \alpha-1}}{1+\left(\frac{R_{W}^{L}}{R^{W}-R_{L}^{W}}\right)^{1 / \alpha-1}}, & R_{W}^{L}\left(R_{L}^{W} / R_{W}^{L}\right)^{\alpha}+R_{L}^{W}<R^{W} .\end{cases}
\end{gathered}
$$

Thus, Theorem 1 allows to find the optimal fraction of time for join access the channels for each fixed fairness coefficient. Figure 1 illustrate that an increase in fairness coefficient leads generally to loss in throughput for one network and to gains in throughput for the other network with $R_{W}=1, R_{W}^{L}=0.7$ and $R_{L}^{W}=$ $0.2,0.3,04$. This puts forward a question: which of this $\alpha$ to assign to make the most fair access the channels for the network. Figure 1 also illustrate that under some condition the optimal fraction of time for join access to the network can be indifferent to fairness coefficient. In the considered example, if $R_{L}^{W}=0.3$ then $q_{\alpha}=5 / 7$ and $q_{\alpha}^{W}=2 / 7$ for any $\alpha$. This puts forward the other question: to find the condition for which the optimal strategy is indifferent to fairness coefficient. 

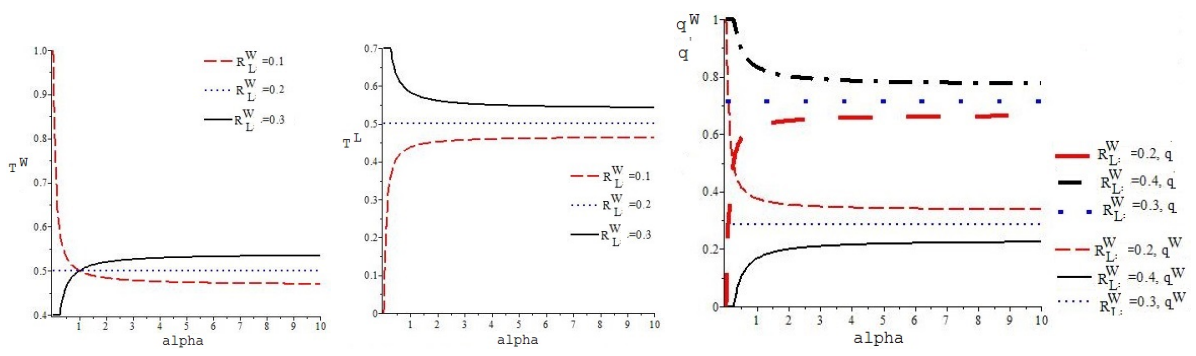

Fig. 1. Wi-Fi throughput (left), LTE-U throughput (middle) and fraction of time for only Wi-Fi network's a access and joint access the channels (right) as functions on fairness coefficient.

\section{Properties of the optimal solution}

In this section, first we find the condition on network parameters when the optimal fair solution is indifferent to fairness coefficient. Second, we simplify the optimal solution for two boundary cases of the fairness coefficient: (a) cooperative solution (i.e., for $\alpha=0$ ) and (b) maxmin solution (i.e., for $\alpha$ tending to infinity).

Corollary 1 The optimal $\alpha$ fair strategy $\boldsymbol{q}_{\alpha}$ does not depend on $\alpha$ if and only if the following condition holds:

$$
R_{L}^{W} \geq R_{W}^{L} \text { and } R_{L}^{W}+R_{W}^{L} \geq R^{W}
$$

Moreover, under condition (4) $\alpha$ fair strategy $\boldsymbol{q}_{\alpha}$ is constant and equals to $(0,1)$.

In particular, if condition (4) holds then maxmin solution and cooperative solution coincide. That is why, below we assume that (4) does not hold. This assumption holds if and only if one of the inequalities in (4) does not hold, i.e.,

$$
R_{L}^{W}<R_{W}^{L} \text { or } R_{L}^{W}+R_{W}^{L}<R^{W} .
$$

Corollary 2 (a) Cooperative solution $\boldsymbol{q}_{0}$ is given as follows:

$$
\left(q_{0}, q_{0}^{W}\right)= \begin{cases}(1,0), & R_{L}^{W}+R_{W}^{L}>R^{W} \\ \text { any }\left(q_{0}, q_{0}^{W}\right) \text { such that } q_{0}+q_{0}^{W}=1 & R_{L}^{W}+R_{W}^{L}=R^{W} \\ (0,1), & R_{L}^{W}+R_{W}^{L}<R^{W}\end{cases}
$$

with payoffs

$$
\left(T_{0}^{L}, T_{0}^{W}\right)=\left\{\begin{array}{ll}
\left(R_{W}^{L}, R_{L}^{W}\right), & R_{L}^{W}+R_{W}^{L}>R^{W} \\
\left(q_{0} R_{W}^{L}, R^{W}-q_{0}\left(R_{L}^{W}-R^{W}\right)\right), & R_{L}^{W}+R_{W}^{L}=R^{W} \\
\left(0, R^{W}\right), & R_{L}^{W}+R_{W}^{L}<R^{W}
\end{array} .\right.
$$

(b) Maxmin solution $\boldsymbol{q}_{\infty}$ is unique and given as follows:

$$
\left(q_{\infty}, q_{\infty}^{W}\right)= \begin{cases}(1,0), & R_{L}^{W} \geq R_{W}^{L} \\ \left(\frac{R^{W}}{R^{W}+R_{W}^{L}-R_{L}^{W}}, \frac{R_{W}^{L}-R_{L}^{W}}{R^{W}+R_{W}^{L}-R_{L}^{W}}\right), & R_{L}^{W}<R_{W}^{L}\end{cases}
$$


with payoffs

$$
\begin{aligned}
& \left(T_{\infty}^{L}, T_{\infty}^{W}\right)= \begin{cases}\left(R_{W}^{L}, R_{L}^{W}\right), & R_{L}^{W} \geq R_{W}^{L}, \\
\left(T_{\infty}, T_{\infty}\right), & R_{L}^{W}<R_{W}^{L}\end{cases} \\
& \text { with } T_{\infty}=\left(R^{W} R_{W}^{L}\right) /\left(R^{W}+R_{W}^{L}-R_{L}^{W}\right)
\end{aligned}
$$

In particular, (a) for $R_{L}^{W}+R_{W}^{L}=R^{W}$, a continuum cooperative solution might arise returning the same total payoff $R^{W}$ to both networks, (b) for $R_{L}^{W}<R_{W}^{L}$ both the Wi-Fi and LTE-U networks have the same maxmin payoff.

\section{Trade-off value for the fairness coefficient}

In previous Section we shown that if condition (5) holds then there is a continuum of fair solution. Namely, a solution $\boldsymbol{q}_{\alpha}$ per an $\alpha$. Then, a question arises: which of these $\left\{\boldsymbol{q}_{\alpha}: \alpha \geq 0\right\}$ is the most fair? To answer on this question we are going to apply Nash bargaining approach [29].

First let us define the feasibility set $\boldsymbol{L}$ of all the fair throughput for the networks, i.e., $\boldsymbol{L}:=\left\{\left(T_{\alpha}^{L}, T_{\alpha}^{W}\right): \alpha \geq 0\right\}$.

Theorem 2 (a) The feasibility set $\boldsymbol{L}$ is a line in plane $\left(T^{L}, T^{W}\right)$ connecting two boundary points $A_{0}:=\left(T_{0}^{W}, T_{0}^{L}\right)$ and $A_{\infty}:=\left(T_{\infty}^{W}, T_{\infty}^{L}\right)$. Moreover, this line can be given in closed form as follows:

$$
T_{\alpha}^{W}=R^{W}-\frac{R^{W}-R_{L}^{W}}{R_{W}^{L}} T_{\alpha}^{L}
$$

with

$$
\left(A_{0}, A_{\infty}\right)= \begin{cases}\left(\left(R_{W}^{L}, R_{L}^{W}\right),\left(T_{\infty}, T_{\infty}\right)\right), & \left(R_{W}^{L}, R_{L}^{W}\right) \in I, \\ \left(\left(0, R^{W}\right),\left(T_{\infty}, T_{\infty}\right)\right), & \left(R_{W}^{L}, R_{L}^{W}\right) \in I I, \\ \left(\left(0, R^{W}\right),\left(R_{W}^{L}, R_{L}^{W}\right)\right), & \left(R_{W}^{L}, R_{L}^{W}\right) \in I I I\end{cases}
$$

where $I:=\left\{\left(R_{W}^{L}, R_{L}^{W}\right): R_{L}^{W}+R_{W}^{L}>R^{W}, R_{L}^{W}<R_{W}^{L}\right\}, I I:=\left\{\left(R_{W}^{L}, R_{L}^{W}\right): R_{L}^{W}+\right.$ $\left.R_{W}^{L}<R^{W}, R_{L}^{W}<R_{W}^{L}\right\}$ and $I I I:=\left\{\left(R_{W}^{L}, R_{L}^{W}\right): R_{L}^{W}+R_{W}^{L}<R^{W}, R_{L}^{W}>R_{W}^{L}\right\}$.

(b) An increase in one expected throughput yields a corresponding decrease in the other throughput.

(c) Each point of the feasibility set $\boldsymbol{L}$ is Pareto optimal.

Second let $\left(T_{d}^{L}, T_{d}^{W}\right)=\left(\min \left\{T_{0}^{L}, T_{\infty}^{L}\right\}, \min \left\{T_{0}^{W}, T_{\infty}^{W}\right\}\right)$ be the point composed by minimal throughput in $\boldsymbol{L}$ of each network. This point can be considered as $a$ disagreement point [29]. Then, the Nash bargaining solution [29] is given as the unique solution of the following optimization problem:

$$
\begin{aligned}
& \text { maximize } N P\left(T^{L}, T^{W}\right):=\left(T^{L}-T_{d}^{L}\right)\left(T^{W}-T_{d}^{W}\right), \\
& \text { subject to }\left(T^{L}, T^{W}\right) \in \boldsymbol{L},
\end{aligned}
$$

where NP is called the Nash product. 
Theorem 3 (a) The bargaining throughput $\left(T^{L}, T^{W}\right)$ e is uniquely given as follows:

$$
T^{L}=T_{d}^{L} / 2+\left(R^{W}-T_{d}^{W}\right) R_{W}^{L} /\left(2\left(R^{W}-R_{L}^{W}\right)\right)
$$

with $T^{W}$ given by (6), and

$$
\left(T_{d}^{L}, T_{d}^{W}\right)= \begin{cases}\left(T_{\infty}, R_{L}^{W}\right), & \left(R_{W}^{L}, R_{L}^{W}\right) \in I \\ \left(0, T_{\infty}\right), & \left(R_{W}^{L}, R_{L}^{W}\right) \in I I \\ \left(0, R_{L}^{W}\right), & \left(R_{W}^{L}, R_{L}^{W}\right) \in I I I\end{cases}
$$

(b) The bargaining value for the fairness coefficient is uniquely defined as follows:

$$
\alpha=1 /\left(1-\ln \left(\frac{R_{W}^{L} R^{W}}{T^{L}\left(R^{W}-R_{L}^{W}\right)}-1\right) / \ln \left(\frac{R_{W}^{L}}{R^{W}-R_{L}^{W}}\right)\right) .
$$

As a numerical illustration, we consider the basic example of a single Wi$\mathrm{Fi}$ and a single LTE-U cell operating on the same channel with $B=1, \alpha_{W}=$ $\beta_{W}=1, \alpha_{L}=\beta_{L}=1, N_{W}=N_{L}=0.1, h_{W W}=h_{W L}=1$ and $h_{L W}=h_{L W}=0.5$. Figure 2 illustrates the bargaining fairness coefficient and the corresponding WiFi throughput and LTE-U throughput as functions of the transmission power $p_{W}$ of the Wi-Fi cell for a fixed transmission power $p_{L}=1$ for the LTE-U cell in the example we considered previously. This figure shows that an increase in the transmission power of the $\mathrm{Wi}-\mathrm{Fi}$ unit leads to an increase in Wi-Fi throughput and to a decrease in the LTE-U throughput. By $(10),\left(T_{d}^{L}, T_{d}^{W}\right)$ has a jump at switching between cases I and II while it is continuous at switching between cases I and II, while $p_{W}$ varies. In the considered basic example, switching between cases I and II, while $p_{W}$ varies, takes place at $p_{W}=0.6$, and this yields a jump increase in Wi-Fi throughput and to a jump decrease in LTE-U throughput. Switching between cases II and III, while varying $p_{W}$, takes place at $p_{W}=1.0$. The bargaining fairness coefficient is decreasing and becomes zero at $p_{W}=0.6$. Thus, at this point the bargaining fair solution becomes a cooperative solution. Further increase in the transmission power leads to an increase in the bargaining fairness coefficient.

Figure 3 illustrates the bargaining fairness coefficient and the corresponding Wi-Fi throughput and LTE-U throughput as functions of the transmission power $p_{L}$ for the LTE- $\mathrm{U}$ cell for a fixed transmission power $p_{W}=1$ associated with the Wi-Fi cell. In this situation, switching between cases II and III can only be observed while $p_{L}$ varies. This switch takes place at $p_{L}=1.0$. It is interesting to note that both cells might achieve a gain resulting from an increase in the LTE-U cell's transmission power, namely, throughput for both systems increase after the switching point $p_{L}=1.0$ as one increases $p_{L}$.

\section{Conclusions}

In this paper, we examined the problem of how Wi-Fi and LTE-U networks should share access to a wireless channel. As a criterion for such joint access, 

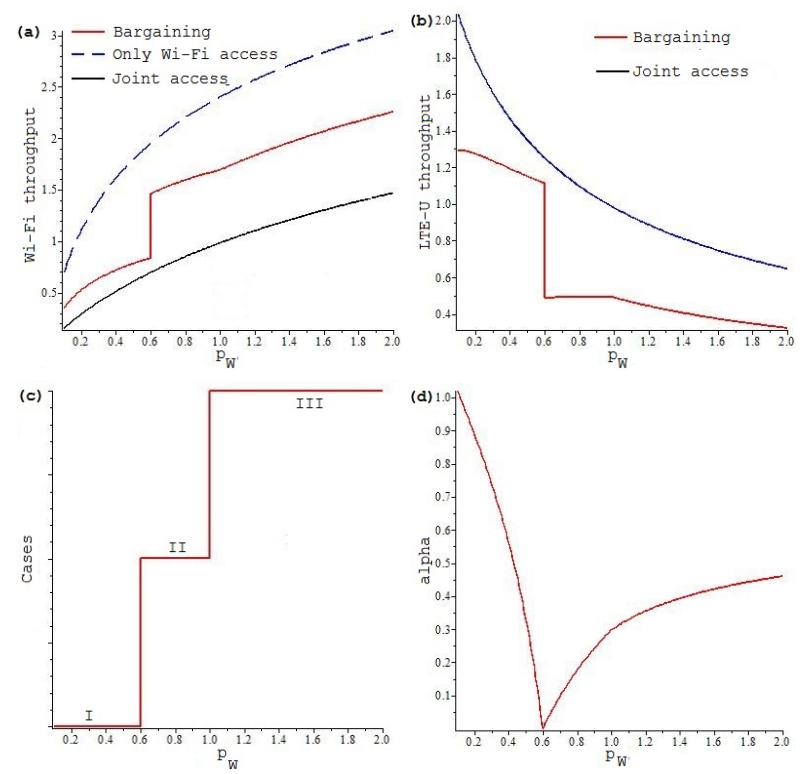

Fig. 2. (a) Wi-Fi throughput, (b) LTE-U throughput, (c) cases and (d) fairness coefficient as functions of the transmission power $p_{W}$ of the Wi-Fi unit with constant transmission power $p_{L}$ for the LTE-U cell with $p_{L}=1$.
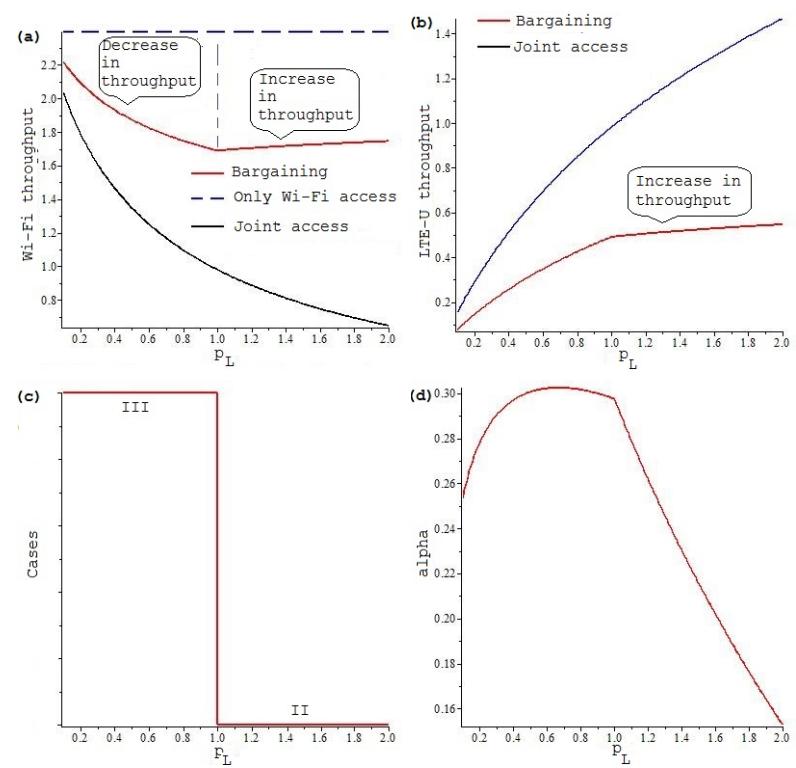

Fig. 3. (a) Wi-Fi throughput, (b) LTE-U throughput, and (c) cases and (d) fairness coefficient as functions of the transmission power $p_{L}$ of the LTE-U cell with constant transmission power $p_{W}$ for the Wi-Fi system with $p_{W}=1$. 
$\alpha$-fairness over the expected throughput was used in this paper since it provides a unifying framework for examining a wide-range of fairness schemes. Following this approach, we arrived at a formal derivation for the continuum of fair protocols (an optimal protocol per $\alpha$ ). We also developed an approach for choosing the unique time-sharing protocol that arises as the solution to the Nash bargaining problem over all the $\alpha$-fair protocols. Under such a protocol, an increase in throughput gains for one network leads to a decrease in performance for the other network, and thus we may consider this protocol as optimal from the point of view of each network. Also, we have shown that such a time-sharing protocol, in spite of the interference between signals, can lead to a gain for both networks under an increase of the transmission power to one of them. Finally, the approach taken in this paper is general and may be applied to examine other problems involving a shared resource.

\section{Appendix}

Proof of Theorem 1. First, let us define the Lagrangian $L_{\omega}(\boldsymbol{q})=v_{\alpha}(\boldsymbol{q})+\omega(1-$ $q^{W}-q$ ), where $\omega$ is a Lagrange multiplier. By Kuhn-Tucker Theorem, for the optimal strategy $\boldsymbol{q}$ (which is a probability vector) the following conditions have to hold:

$$
\begin{gathered}
\frac{R^{W}}{\left(q^{W} R^{W}+q R_{L}^{W}\right)^{\alpha}} \begin{cases}=\omega, & q^{W}>0, \\
\leq \omega, & q^{W}=0,\end{cases} \\
\frac{R_{L}^{W}}{\left(q^{W} R^{W}+q R_{L}^{W}\right)^{\alpha}}+\frac{R_{W}^{L}}{\left(q R_{W}^{L}\right)^{\alpha}} \begin{cases}=\omega, & q>0, \\
\leq \omega, & q=0 .\end{cases}
\end{gathered}
$$

Hence, the boundary strategy $\boldsymbol{q}=(1,0)$ cannot be optimal. Thus only two cases have to be considered (a) $q^{W}=0$ and $q>0$, and (b) $q^{W}>0$ and $q>0$.

(a) Let $q^{W}=0$ and $q>0$. Then, $q=1$, and, by (12) and (13),

$$
R^{W} /\left(R_{L}^{W}\right)^{\alpha} \leq \omega=R_{L}^{W} /\left(R_{L}^{W}\right)^{\alpha}+R_{W}^{L} /\left(R_{W}^{L}\right)^{\alpha} .
$$

This is equivalent to the upper condition in (2) and (3), and (a) follows.

(b) Let $q^{W}>0$ and $q>0$. normalsize Then, by (12) and (13),

Thus,

$$
\frac{R^{W}}{\left(q^{W} R^{W}+q R_{L}^{W}\right)^{\alpha}}=\frac{R_{L}^{W}}{\left(q^{W} R^{W}+q R_{L}^{W}\right)^{\alpha}}+\frac{R_{W}^{L}}{\left(q R_{W}^{L}\right)^{\alpha}} .
$$

$$
q^{W} R^{W}+q R_{L}^{W}=q R_{W}^{L}\left(\left(R^{W}-R_{L}^{W}\right) / R_{W}^{L}\right)^{1 / \alpha} .
$$

Since $q+q^{W}=1$, (14) implies that $q$ and $q^{W}$ has to be given by bottom line of (2) and (3). While the condition $0<q, q^{W}<1$ is equivalent to the bottom condition in (2) and (3), and the result follows.

Proof of Corollary 1. Due to if $R_{L}^{W}<R_{W}^{L}$ then $\left(R_{L}^{W} / R_{W}^{L}\right)^{\alpha}<1$ for and $\alpha \geq 0$, and if $R_{L}^{W}>R_{W}^{L}$ then $\left(R_{L}^{W} / R_{W}^{L}\right)^{\alpha}>1$ for and $\alpha \geq 0$, the result follows from Theorem 1 . 
Proof of Theorem 2. Recall that $T^{W}=q^{W} R^{W}+q R_{L}^{W}$ and $T^{L}=q R_{W}^{L}$. This, jointly with the fact that $\boldsymbol{q}$ is a probability vector, implies (6), and the result follow.

Proof of Theorem 3. Substituting (6) into Nash product given by (8) turns (8) into a maximization problem for a quadratic polynom on $T^{L}$, and (9) follows. Since $R_{W}^{L} q_{\alpha}=T^{L}$, by (3), the bargaining value for the fairness coefficient is uniquely defined by (11), and the result follows.

\section{References}

1. Barbagallo, P., McElgunn, T.: 3gpp set to study lte over unlicensed technology, sowing new spectrum battles. Bloomberg BNA (September 10, 2014) http://www . bna.com/3gpp-set-study-n17179894699/.

2. Cavalcante, A.M., Almeida, E., Vieira, R.D., Choudhury, S., Tuomaala, E., Doppler, K., anf R.C.D Paiva, F.C., Abinader, F.: Performance evaluation of LTE and Wi-Fi coexistence in unlicensed bands. In: IEEE Vehicular Technology Conference (VTC Spring). (2013) 1-6

3. Abinader, F.M., Almeida, E.P.L., Chaves, F.S., Cavalcante, A.M., Vieira, R.D., Paiva, R.C.D., Sobrinho, A.M., Choudhury, S., Tuomaala, E., Doppler, K., Sousa, V.A.: Enabling the coexistence of LTE and Wi-Fi in unlicensed bands. IEEE Communications Magazine 52 (2014) 54-61

4. Liu, F., Bala, E., Erkip, E., Yang, R.: A framework for femtocells to access both licensed and unlicensed bands. In: 9th International Symposium on Modeling and Optimization in Mobile, Ad Hoc and Wireless Networks (WiOpt). (2011) 407-411

5. Kwan, R., Pazhyannur, R., Seymour, J., Chandrasekhar, V., Saunders, S.R., Bevan, D., Osman, H., Bradford, J., Robson, J., Konstantinou, K.: Fair co-existence of licensed assisted access LTE (LAA-LTE) and Wi-Fi in unlicensed spectrum. In: 7th Computer Science and Electronic Engineering Conference (CEEC). (2015) 13-18

6. Hajmohammad, S., Elbiaze, H., Ajib, W.: Fine-tuning the femtocell performance in unlicensed bands: Case of wifi co-existence. In: Int. Wireless Communications and Mobile Computing Conference (IWCMC). (2014) 250-255

7. Yun, S., Qiu, L.: Supporting WiFi and LTE co-existence. In: IEEE Conference on Computer Communications (INFOCOM). (2015) 810-818

8. Liu, F., Erkip, E., Beluri, M., Yang, R.: Small cell traffic balancing over licensed and unlicensed bands. IEEE Trans. on Vehicular Technology 64 (2015) 5850-5865

9. Hajmohammad, S., Elbiaze, H.: Unlicensed spectrum splitting between femtocell and wifi. In: IEEE Int. Conference on Communications (ICC). (2013) 1883-1888

10. Jian, Y., Shih, C.-F., Krishnaswamy, B., Sivakumar, R.: Coexistence of Wi-Fi and LAA-LTE: Experimental evaluation, analysis and insights. In: IEEE International Conference on Communications (ICC). (2015) 10387-10393

11. Cano, C., Leith, D.: Coexistence of WiFi and LTE in unlicensed bands: A proportional fair allocation scheme. In: IEEE International Conference on Communications (ICC). (2015) 10350-10355

12. Sagari, S., Seskar, I., Raychaudhuri, D.: Modeling the coexistence of lte and wifi heterogeneous networks in dense deployment scenarios. In: IEEE International Conference on Communications (ICC). (2015) 10363-10368

13. Raychaudhuri, D., Baid, A.: NASCOR: Network assisted spectrum coordination service for coexistence between heterogeneous radio systems. IEICE Transactions on Communications E97-B (2014) 251-260 
14. Sagari, S., Baysting, S., Sahay, D., Seskar, I., Trappe, W., Raychaudhuri, D.: Coordinated dynamic spectrum management of LTE-U and Wi-Fi networks. In: IEEE International Conference on Dynamic Spectrum Access Network (DySPAN). (2015)

15. Garnaev, A., Sagari, S., Trappe, W.: Fair channel sharing by Wi-Fi and LTE-U networks with equal priority. In Noguet, D., Moessner, K., Palicot, J., eds.: Cognitive Radio Oriented Wireless Networks. Volume 172 of Lecture Notes of the Institute for Computer Sciences, Social Informatics and Telecommunications Engineering., Springer (2016) 91-103

16. Yerramalli, S., Jain, R., Mitra, U.: Coalitional games for transmitter cooperation in mimo multiple access channels. IEEE Trans. on Signal Processing 62 (2014) $757-771$

17. Garnaev, A., Trappe, W.: A bandwidth monitoring strategy under uncertainty of the adversary's activity. IEEE Trans. Information Forensics and Security 11 (2016) 837-849

18. Garnaev, A., Trappe, W.: One-time spectrum coexistence in dynamic spectrum access when the secondary user may be malicious. IEEE Transactions on Information Forensics and Security 10 (2015) 1064-1075

19. Marina, N., Arslan, G., Kavcic, A.: A power allocation game in a four node relay network: An upper bound on the worst-case equilibrium efficiency. In: International Conference on Telecommunications (ICT). (2008) 1-6

20. Altman, E., Avrachenkov, K., Garnaev, A.: Transmission power control game with SINR as objective function. In Altman, E., Chaintreau, A., eds.: Network Control and Optimization. Volume 5425 of LNCS., Springer (2009) 112-120

21. Altman, E., Avrachenkov, K., Garnaev, A.: Closed form solutions for water-filling problems in optimization and game frameworks. Telecommunication Systems $\mathbf{4 7}$ (2011) 153-164

22. Huaizhou, S., Prasad, R.V., Onur, E., Niemegeers, I.G.M.M.: Fairness in wireless networks: Issues, measures and challenges. IEEE Communications Surveys \& Tutorials 16 (2014) 5-24

23. Mo, J., Walrand, J.: Fair end-to-end window-based congestion control. IEEE/ACM Trans. on Networking 8 (2000) 556-567

24. Garnaev, A., Trappe, W.: Bargaining over the fair trade-off between secrecy and throughput in OFDM communications. IEEE Transactions on Information Forensics and Security 12 (2017) 242-251

25. Militano, L., Niyato, D., Condoluci, M., Araniti, G., Iera, A., Bisci, G.M.: Radio resource management for group-oriented services in LTE-A. IEEE Transactions on Vehicular Technology 64 (2015) 3725-3739

26. Garnaev, A., Trappe, W., Petropulu, A.: Bargaining over fair performing dual radar and communication task. In: 50th Asilomar Conference on Signals, Systems, and Computers, Pacific Grove, CA (2016) 47-51

27. Altman, E., Avrachenkov, K., Garnaev, A.: Fair resource allocation in wireless networks in the presence of a jammer. Performance Evaluation 67 (2010) 338-349

28. Garnaev, A., Trappe, W.: Fair resource allocation under an unknown jamming attack: a Bayesian game. In: IEEE Workshop on Information Forensics and Security (WISP), Atlanta, GA (2014) 227-232

29. Fudenberg, D., Tirole, J.: Game theory. MIT Press, Boston, MA (1991) 